(C) Copyright 2020: Editum. Servicio de Publicaciones de la Universidad de Murcia. Murcia (Spain) ISSN print edition: 0212-9728. ISSN online edition (http://revistas.um.es/analesps): 1695-2294. Online edition License Creative Commons 4.0: BY-SA

\title{
Identificación de patrones instruccionales de mediación relacionados con progresos en comprensión lectora en contextos de desventaja sociocultural
}

\author{
Tricia Mardones ${ }^{1}$, Juan-José Navarro ${ }^{2}$ y Leandro Zamorano ${ }^{3}$ \\ 1 Universidad Metropolitana de Ciencias de la Educación, UMCE (Chile). \\ 2 Universidad de Zaragoza (España). \\ 3 Universidad Autónoma de Chile (Chile).
}

\begin{abstract}
Resumen: Identificar factores instruccionales específicos relacionados con progresos en comprensión lectora es esencial para optimizar el aprendizaje y orientar la intervención, especialmente en contextos de desventaja sociocultural o dificultades de aprendizaje. Este estudio pretende identificar patrones instruccionales de mediación eficaces en la explicación del progreso en comprensión. Se grabaron, transcribieron y analizaron sistemáticamente 82 sesiones-clase, y se registraron pautas de mediación relativas a diferentes procesos lectores. Los patrones de mediación fueron analizados mediante codificación y categorización del contenido con el programa Maxqdea-7. Fueron consideradas 19 categorías, que aglutinaron 178 unidades de información significativas (pautas/conductas de mediación). La muestra estuvo compuesta por 21 docentes y 821 estudiantes de tercero a octavo grado de Educación Básica de escuelas públicas de Chile ubicadas en contextos deprivados socialmente. El progreso lector se midió con la prueba estandarizada CL-PT, aplicada al principio y al final del curso escolar. Los resultados permitieron identificar pautas específicas de mediación significativas en la explicación del progreso, especialmente el patrón M_17 (Mediación del uso de estrategias para la activación e integración de conocimientos previos) explicaba el $26 \%$ de la varianza $\left(r=.55, p<.01 ; R^{2 a j u s t a d o}=.26, F_{(1,19)}=8.19, p=.01\right)$. Se discuten las implicaciones educativas de estos resultados.

Palabras clave: patrones de mediación; pautas docentes; rendimiento lector; comprensión lectora; desventaja sociocultural.
\end{abstract}

Title: Identifying instructional mediation patterns related with progress made in reading comprehension in socio-cultural disadvantaged contexts. Abstract: Identifying specific instructional factors related to advances made in reading comprehension is essential for optimizing learning and for guiding interventions, especially in socio-cultural disadvantaged contexts or with learning difficulties. This study intends to identify efficient instructional mediation patterns to explain progress made in comprehension. Eighty-two sessions-classes were systematically recorded, transcribed and analyzed, and the mediation patterns related to different reading processes were recorded. Mediation patterns were analyzed by coding and classifying contents with the Maxqdea-7 program. Nineteen categories were considered, which included 178 significant information units (mediation patterns/conducts). The sample was made up of 21 teachers and 821 students of years 3 to 8 of Basic Education in public Chilean schools from socially deprived contexts. Reading progress was measured by the standard CL-PT test, which was applied at the beginning and end of the academic year. The results identified specific significant mediation patterns of progress, especially pattern M_17 (Mediation of using strategies for activating previous knowledge, and for integrating knowledge and experiences with new contents) which explained $26 \%$ of variance $\left(r=.55, p<.01\right.$; adjusted $R^{2}=.26, F_{(1,19)}=8.19, p=$ $.01)$. The educational implications of these results are discussed.

Keywords: mediation patterns; teaching patterns; reading performance; reading comprehension; socio-cultural disadvantage.

\section{Introducción}

El fenómeno de la estratificación socioeconómica y su relación con el progreso y rendimiento escolar, constituyen una realidad difícil de soslayar en muchos países, al tiempo que una preocupación constante para educadores y gestores públicos. Esta realidad ha sido estudiada en Chile en relación a la implementación de políticas públicas compensatorias, como el programa P-900 o la Ley N 20248 (Ley de Subvención Escolar Preferencial, 2008), así como en relación a desvelar la incidencia diferencial de los distintos factores que la explican (Cadiz \& Martinic, 2007). También los resultados de pruebas de diagnóstico educativo nacionales e internacionales, como los ofrecidos por el Sistema de Medición de la Calidad en la Educación (SIMCE), y por los programas internacionales PIRLS (Progress in International Reading Literacy Study) y PISA (Programme for International Student Assessment), muestran la estrecha conexión existente entre el nivel socioeconómico familiar y el rendimiento académico. Los resultados SIMCE de estudiantes chilenos en comprensión lectora, mostraron entre los años 2014-2015 un aumen-

* Correspondence address [Dirección para correspondencia]:

Juan-José Navarro. Facultad de Educación, Departamento de Psicología y Sociología. Universidad de Zaragoza. C/ Pedro Cerbuna, 12, 50009, Zaragoza (Spain). E-mail: jinh@unizar.es

(Artículo recibido: 18-10-2018, revisado: 10-12-2018, aceptado: 12-10-2019) to de la brecha de rendimiento entre grupos socioeconómicos altos y bajos (Agencia de Calidad de la Educación, 2016a), revelando ausencia de progreso e incluso retrocesos en los promedios obtenidos por los grupos socioeconómicos medio-bajo y bajo respectivamente, con puntuaciones significativamente por debajo del resto de grupos sociales. Por su parte, los resultados obtenidos en las pruebas PIRLS y PISA evidenciaron diferencias promedio significativas (95 y 96 puntos respectivamente) entre estudiantes de altos y bajos recursos (Agencia de Calidad de la Educación, 2016b). Los últimos resultados disponibles en ambas pruebas muestran, a pesar de los avances logrados, que las puntuaciones obtenidas en comprensión lectora se encuentran significativamente por debajo del promedio internacional, situándose en niveles bajos o muy bajos de comprensión entre un $28,4 \%$ y un $39 \%$ de los estudiantes evaluados.

La investigación también revela a nivel internacional, que estudiantes en situación de desventaja sociocultural y económica presentan habitualmente peores resultados en comprensión lectora que aquellos que provienen de sectores socioeconómicos altos (García-Crespo, Galián, FernándezAlonso, \& Muñiz, 2019). Las condiciones de tipo estructural que caracterizan la deprivación sociocultural, desencadenarían una serie de factores que podrían explicar desde una óptica más próxima el menor rendimiento académico de estos estudiantes. Entre estos factores, destacan: (a) una menor y más empobrecida exposición a estímulos y experiencias de 
aprendizaje en relación con la cultura escrita (Urquijo, García-Coni, \& Fernandes, 2015); (b) un uso empobrecido del lenguaje, con escasa presencia de formas y estructuras complejas, y una mayor carencia de conocimientos específicos en comparación con sus compañeros (Fernald, Marchman, \& Weisleder, 2013; Kieffer, 2012); (c) la presencia de problemas para atender selectivamente, dificultando de este modo el filtrado de información relevante, e influyendo negativamente en el proceso de inhibición latente (Schibli, Wong, Hedayati, \& D’Angiulli, 2017); y (d) dificultades de índole emocional y conductual, ya sean de carácter internalizante, como ansiedad, bajas expectativas, o patrones atribucionales de éxito o fracaso desajustados; o externalizante, como disrupción, hiperactividad, o agresividad (Flouri, Midouhas, Joshi, \& Sullivan, 2016; Schibli et al., 2017).

Algunos estudios están apuntando a la incidencia significativa que pueden llegar a tener variables relativas a la organización escolar o las dinámicas de participación, así como otras relativas a los procesos de enseñanza-aprendizaje, como el clima y la gestión del aula, la estructura de participación de los distintos actores que intervienen, o los procesos de mediación que los docentes ponen en práctica para promover los aprendizajes (Boardman, Boelé, \& Klingner, 2018; Flecha, Garcia, Gomez, \& Latorre, 2009; GarcíaCrespo et al., 2019; Hou, 2018; Navarro \& Mora, 2013; Resing, 2013; Valls, Soler, \& Flecha, 2008). A la luz de los datos ofrecidos por el programa PIRLS, García-Crespo et al. (2019) llevaron a cabo un estudio que analizaba diversos factores que podrían incidir en la capacidad de los estudiantes para obtener buenos resultados en la escala de comprensión lectora de la prueba PIRLS 2016 en contextos de desventaja socioeconómica y cultural. Los datos analizados $(N=$ 117539 estudiantes de 23 países de la Unión Europea), asumiendo las diferencias entre países, mostraron factores relativos a la escuela y la historia escolar, como la disciplina instaurada en el centro (8 países), haber tenido tareas tempranas de competencia lectora (11 países), o el énfasis académico de la escuela (4 países), que incrementaron significativamente la probabilidad de que estudiantes con bajo nivel socioeconómico y cultural (último cuartil) obtuvieran resultados altos (primer cuartil) en la prueba. En este sentido, si bien nadie discute la relevancia de variables estructurales en los resultados académicos, algunos factores claves asociados al éxito y eficacia escolar podrían estar relacionados con la implementación y consolidación de prácticas educativas que supongan una transformación efectiva a nivel escolar y de aula (Valls et al., 2008). De este modo, se asume que la escuela puede promover cambios y lograr mejorar el rendimiento, incluso en sectores sociales desaventajados (Flecha et al., 2009; García-Crespo et al., 2019; Muijs, 2003).

Lo planteado anteriormente vendría reforzado por una perspectiva ecológico-sistémica que plantea que los factores de cambio no ejercerían su influencia en modo lineal, sino que incidirían en modo interactivo y contextualizado. De este modo, la actuación y los cambios promovidos en algún componente del sistema, pueden incidir en el resto mediante la continua interacción entre estos componentes, situados en un contexto determinado (Bronfenbrenner, 1987). De este modo, las actuaciones docentes objeto de análisis se entienden como un componente más del sistema escuela en el que es posible introducir cambios a raíz de la identificación de pautas de mediación eficaces y claves para explicar mejoras significativas en los estudiantes (Rueda, 2013). Así mismo, desde esta perspectiva, se considera posible identificar patrones docentes de mediación en actividades específicas como la lectura que pueden estar mediando y promoviendo cambios y mejoras significativas en los estudiantes (Navarro, Mora, Lama, \& Molina, 2014). Dentro de este marco, el presente estudio pretende identificar y analizar estos patrones docentes que actuarían como factores decisivos en las mejoras obtenidas aún en contextos de deprivación sociocultural (Bellei, Valenzuela, Vanni, \& Contreras, 2014).

\section{Mediación y patrones instruccionales}

Investigaciones en el ámbito del lenguaje en educación básica, plantean que existen rasgos compartidos en las acciones instruccionales impartidas por los docentes (Preiss, 2009). Este autor analizó el proceso de mediación a partir del concepto de Patrones Instruccionales, entendidos como aquellos patrones de mediación más utilizados por los docentes en un contexto educativo y cultural determinado. Estos patrones instruccionales actuarían como referentes que orientan el trabajo docente y a su vez condicionan las posibilidades de innovación pedagógica. En cuanto a las conclusiones observadas, los patrones instruccionales encontrados se caracterizaron por el predominio de interacciones pedagógicas donde el rol docente estaba centrado en el control externo y el seguimiento a la adquisición de información por parte del estudiante. En este sentido las oportunidades brindadas por los docentes para el desarrollo de procesos de autorregulación o de carácter metacognitivo fueron muy escasas (Preiss, 2009; Preiss et al., 2014). Sin embargo, distintos estudios han mostrado la relevancia de los procesos de control y autorregulación en la comprensión lectora (Navarro et al., 2014; Souvignier \& Mokhlesgerami, 2006; Thiede, Griffin, Wiley, \& Redford, 2009). La mayoría de estos estudios enfatiza la mediación de habilidades metacognitivas en los procesos de intervención, esencialmente habilidades de autorregulación cognitiva, aunque también señalan la relevancia de integrar los aspectos socioafectivos y motivacionales, relacionando las mejoras observadas con el incremento en las competencias relacionadas precisamente con estas habilidades.

En línea con las ideas expuestas, diversos estudios han evidenciado mejoras significativas en estudiantes con dificultades de aprendizaje, discapacidad o desventaja sociocultural tras su participación en estudios instruccionales, programas de enriquecimiento cognitivo y procedimientos de evaluación dinámica caracterizados por el uso de diversas formas de mediación (Aguilera \& Mora, 2004; Beckmann, Beckmann, \& Elliott, 2009; Carlson \& Wiedl, 2000; Navarro et al., 2014; Resing, Tunteler, de Jong, \& Bosma, 2009; Resing 
\& Elliott, 2011). En lo que respecta a la evaluación, estos procedimientos han sido desarrollados en su mayor parte en relación al trabajo específico con estos sujetos, y la relevancia de su aplicación parece ser mayor precisamente en estos casos. Así mismo, estos procedimientos han tratado de ofrecer información adicional a la aportada por pruebas de evaluación convencionales (Sternberg \& Grigorenko, 2002). Esta información adicional se ha concretado a veces en analizar el proceso de aprendizaje y las dificultades encontradas por el estudiante durante el mismo, así como en el análisis del proceso de mediación o interacción mediada característico de estos modelos. En definitiva, el estudio de los mecanismos que explican las mejoras que pueden observarse durante la aplicación de procedimientos dinámicos de evaluación, y a través de los cuales un estudiante optimizaría su aprendizaje, puede ser significativo para orientar la intervención, así como los procesos de formación docente (Aguilera \& Mora, 2004; Grigorenko, 2009; Roskos, Moe, \& Rosemary, 2017). De este modo, el aplicador de estos procedimientos, a través de la puesta en práctica de pautas de interacción mediada, ofrecería herramientas de control y regulación de la actividad con el objetivo de observar y valorar el grado de incorporación de estas herramientas por parte del estudiante durante la resolución de la tarea. Así mismo, a través de estas pautas, ejerce una mediación entre las competencias actuales del estudiante y aquellas necesarias para realizar con éxito la tarea propuesta, tratando de obtener información valiosa que oriente la intervención. De este modo, los procesos de análisis de la aplicación contextualizada de algunos de estos procedimientos, han facilitado la extracción de una serie de pautas de interacción que los aplicadores han puesto en práctica durante el desarrollo de las sesiones y que habrían resultado claves en la obtención de las mejoras observadas. En algunos casos, los procesos de formación necesarios para la implementación de estos procedimientos, han incorporado entrenamiento específico en el uso de estas pautas de interacción (Aguilera \& Mora, 2004; Navarro et al., 2014; van Driel, Slot, \& Bakker, 2018).

En este sentido, el análisis del proceso de enseñanzaaprendizaje y la caracterización de los patrones de mediación utilizados por los docentes en el presente estudio, que se hubieran revelado claves en la obtención de mejoras observadas, podría ofrecer información adicional y cualitativa orientada tanto a la intervención con los estudiantes, como a la mejora del proceso de formación docente. Recoger aquellas experiencias exitosas de progreso en contextos de desventaja sociocultural, desde el análisis de la práctica docente, permitiría identificar posibles patrones eficaces de mediación. Entendemos que identificar estos patrones de mediación supone situar el foco en procesos de actuación eficientes. Así mismo, analizar lo que hacen los docentes en el aula para promover la comprensión en sus estudiantes, implica partir del análisis de la práctica educativa real para poder establecer procesos graduales de formación continua con mayores garantías de éxito (Sánchez, 2010). De este modo, la información aportada permitiría orientar procesos de formación ini- cial y, sobre todo, de desarrollo profesional docente en relación a la enseñanza-aprendizaje de la lectura durante la enseñanza básica en contextos de especial dificultad.

\section{Objetivos e hipótesis de trabajo}

El presente estudio tiene como objetivo identificar patrones docentes de mediación desplegados en actividades específicas de lectura, que habrían actuado como factores clave en el progreso obtenido por estudiantes en situación de desventaja sociocultural. En este sentido, se pretende identificar aquellos patrones de mediación utilizados por los docentes durante las sesiones analizadas, que muestren una relación estadísticamente significativa con el progreso obtenido en comprensión lectora por los estudiantes, medido este a través de la diferencia entre las evaluaciones inicial y final. A modo de hipótesis de trabajo, esperábamos que las puntuaciones obtenidas por los estudiantes con mayor progreso en lectura, estuvieran significativamente relacionadas con una mayor utilización de patrones de mediación de carácter metacognitivo y autorregulatorio, así como de aquellos dirigidos a procesos de ajuste personal-social.

\section{Método}

\section{Diseño de investigación y procedimiento}

El diseño es no experimental, adoptando una estrategia de investigación asociativa. Se trata de un estudio predictivo de carácter transversal (Ato, López, \& Benavente, 2013), en el que los participantes fueron seleccionados intencionalmente en función de las características de los establecimientos educativos en los que desarrollan su actividad (situación de vulnerabilidad social). Esencialmente, analiza la relación entre los patrones docentes de mediación utilizados en el transcurso de actividades de lectura en distintos grupos-clase y el progreso lector observado. Con este objetivo se ha desarrollado un estudio en tres fases durante un curso escolar: (a) evaluación inicial, (b) observación, registro audiovisual e identificación de conductas de mediación, y (c) evaluación final. Entre la evaluación inicial y final del rendimiento lector transcurrieron 6 meses. Durante la fase de observación, se han registrado sistemáticamente las pautas docentes de mediación en relación a distintos procesos implicados en la lectura. El análisis de la información proporcionada contempla partir de los distintos grupos-clase considerados para el análisis de los patrones de mediación, en función de la obtención de distintos grados de cambio entre las puntuaciones iniciales y finales (Aguilera y Mora, 2004). Tomados dichos grupos como sujetos de investigación, hemos propuesto la hipótesis general de que la calidad de los patrones de mediación utilizados por cada docente, contribuiría a explicar significativamente la diferencia de resultados. Concretamente, esperábamos que las puntuaciones obtenidas por los estudiantes con mayor progreso en lectura se relacionaran significativamente con la puesta en práctica de mediaciones de 
carácter regulatorio y metacognitivo, así como de aquellas de carácter más cálido, dirigidas a procesos de ajuste personalsocial. Con el fin de verificar esta hipótesis se han utilizado instrumentos de observación y análisis de la actividad y el discurso desarrollados en el aula, a raíz de grabaciones en video de distintas sesiones de trabajo.

Se mantuvieron distintas reuniones informativas con docentes y familias, donde se expusieron los objetivos esenciales del estudio y se solicitó el consentimiento informado. Se obtuvo asimismo información de los establecimientos educativos en relación al nivel socioeconómico.

\section{Participantes}

La muestra estuvo compuesta por 21 grupos-clase con un total de 821 estudiantes ( $45.5 \%$ niñas y $54.5 \%$ niños; $M$ edad $=10.37, D T=2.06)$ de $3^{\circ}$ a $8^{\circ}$ de Educación Básica de seis escuelas públicas de Santiago de Chile, situadas en contextos de alta vulnerabilidad social según el índice de la Junta Nacional de Auxilio Escolar y Becas (JUNAEB, 2012) y los datos de SIMCE 2012-2016 (Agencia de Calidad de la Educación, 2016a). Los docentes (12 mujeres y 9 hombres) eran especialistas del área de Lenguaje e Historia. La mayoría eran docentes experimentados. Los más jóvenes contaban al menos con cuatro años de experiencia.

\section{Instrumentos}

Lectura y comprensión. Se ha utilizado la prueba de Comprensión Lectora y Producción de textos CL-PT, (Medina,
Gajardo, \& Fundación Arauco, 2010). La prueba CL-PT está validada en Chile y su uso es generalizado en el contexto escolar. Está prueba contiene tests específicos para cada curso escolar. Los rasgos que evalúa son: comprensión de estructuras textuales, comprensión literal, comprensión inferencial, comprensión crítica, comprensión metacognitiva, reorganización de la información, y manejo del código. La prueba está constituida por entre 15 y 16 ítems en función de los cursos evaluados, entre los cuales se distribuyen los rasgos anteriormente mencionados. A su vez, la prueba CL-PT establece 5 niveles de desempeño en función de la puntuación obtenida y el porcentaje de logro total: (1) No desarrollado; (2) Emergente; (3) En desarrollo; (4) Desarrollo satisfactorio; y (5) Muy desarrollado.

Instrumentos para la fase de observación. Se ha utilizado el programa de análisis cualitativo Maxqdea-7. Se registraron audiovisualmente en promedio 4 sesiones de clase de cada uno de los profesores participantes. El conjunto de sesiones analizadas ha sido de 82 , con un promedio de 70.23 (DT $=$ 13.68) minutos por sesión. Finalmente, el total de horas de grabación analizadas ha sido de 98.5. Estas sesiones fueron posteriormente transcritas y analizadas mediante el citado programa (Fig. 1), con el fin de facilitar la codificación y categorización de los segmentos de información significativos e identificar los patrones de mediación en relación con los diferentes procesos implicados en la lectura.

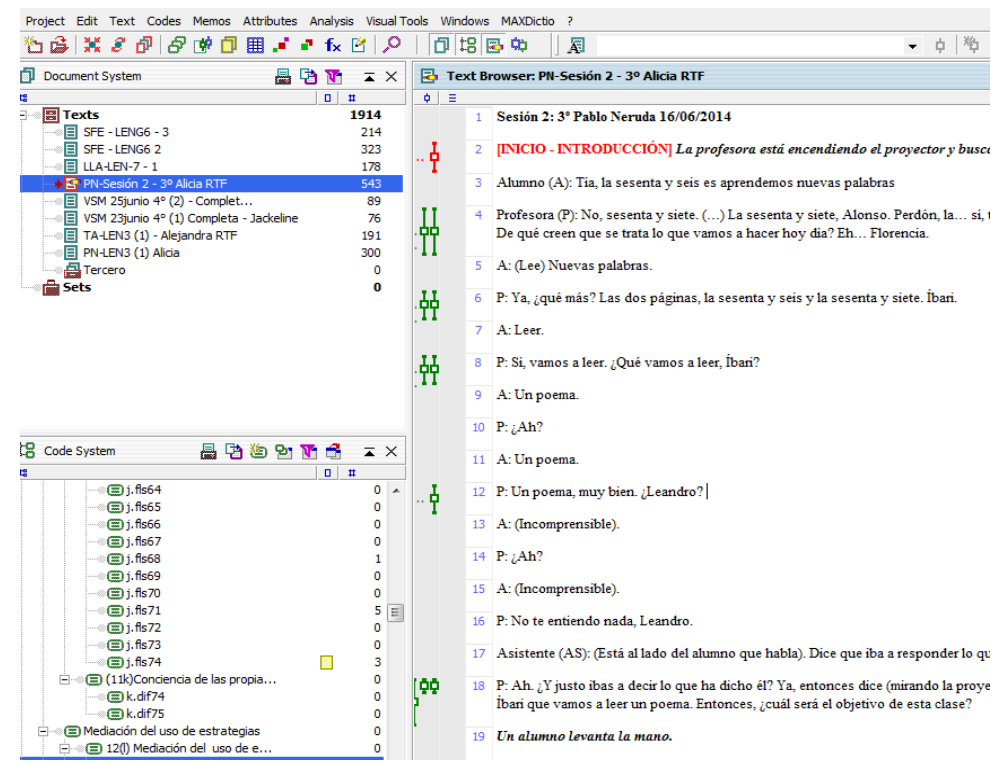

Figura 1 Identificación de pautas de mediación en el programa Maxqdea.

El sistema de categorías utilizado se estructuró en tres bloques o dimensiones: (a) mediación de aspectos generales, (b) mediación del conocimiento metacognitivo y uso de estrategias, y (c) mediación del ajuste personal-social (Tabla 1). Fueron consideradas inicialmente 19 categorías, que aglutina- ron a su vez 178 unidades de información significativas (pautas/conductas de mediación). Los procesos implicados en la lectura y los patrones de mediación que han sido utilizados en el presente estudio, fueron desarrollados en una investigación previa (Navarro \& Mora, 2011; 2013; Navarro et al., 
2014), en la cual se analizó la implementación de un dispositivo de evaluación dinámica de procesos lectores (EDPL). Los procesos contemplados se encuentran en consonancia con los mayormente citados en la literatura (Beker, Jolles, Lorch, \& van den Broek, 2016; Compton et al., 2010; Graesser, Singer, \& Trabasso, 1994; Hacker, 1998; León, Escudero, \& Olmos, 2012; Rueda, 2013; Sánchez, 2010). El proceso de mediación propuesto en el dispositivo EDPL incluía un conjunto de pautas (no estandarizadas), tales como guía metacognitiva, sugerencias graduadas, o feedback, que estaban asociadas específicamente a cada una de las tareas. Los evaluadores debían aplicar estas pautas con el fin de evaluar el proceso de aplicación y el grado y tipo de apoyo que necesitaba el estudiante durante la resolución de las tareas, y también con el fin de observar y evaluar en qué medida el alumno incorporaba algunas de las estrategias utilizadas en el proceso de mediación. En este sentido, la unidad de análisis básica ha sido la actividad de mediación (ayudas) desarrollada por el docente.

Tabla 1. Sistema de categorías utilizado inicialmente para la identificación de los patrones de mediación. Mediación de aspectos generales

1 (a) Profundización en la respuesta o en su justificación, haciendo nuevas preguntas o cuestionando sus respuestas

2 (b) Ampliación de la actividad mediante la realización de tareas relacionadas que surgen del propio alumnado o del profesor/evaluador

3 (c) Establecimiento de apoyo en la secuenciación, comprensión o realización de la actividad

4 (d) Recapitulación conjunta del trabajo realizado anteriormente con el objetivo de retomarlo

5 (e) Mediación de la discusión y la reflexión sobre contenidos, elementos, estrategias y procesos

6 (f) Repetición de una actividad para poder poner en práctica las estrategias trabajadas y para poder evaluar su incorporación a la ejecución de la actividad por parte del alumno

\section{Mediación del conocimiento metacognitivo y del uso de estrategias}

7 (g) Conciencia de lo que sabe sobre elementos estructurales y tipologías de textos

8 (h) Conciencia sobre las propias estrategias de comprensión, de cuándo, cómo y por qué son útiles

9 (i) Conciencia de lo que sabe acerca del contenido de los textos trabajados o de una actividad

10 (j) Conciencia de la estructura fonológica, léxica y sintáctica del lenguaje

$11(\mathrm{k})$ Conciencia de las propias dificultades relacionadas con la lectura

12 (l) Mediación del uso de estrategias de planificación

13 (m) Mediación del uso de estrategias de supervisión

14 (n) Mediación del uso de estrategias de autoevaluación

15 (ñ) Mediación del uso de estrategias de transferencia y generalización

16 (o) Mediación del uso de estrategias de integración textual

17 (p) Mediación del uso de estrategias para la activación de conocimientos previos y la integración de conocimientos y experiencias con nuevos contenidos

Mediación de procesos de ajuste personal-social

18 (q) Mediación de la motivación, la actitud y el interés con relación a la lectura

19 (r) Mediación de la participación y el apoyo entre los componentes del grupo

\section{Análisis de datos}

Parte de los análisis se llevaron a cabo con el programa estadístico SPSS/PC (versión 21). Se realizaron análisis jerárquicos de regresión múltiple, en los que se situaron como variables dependientes las puntuaciones de mejora de los estudiantes en lectura, obtenidas de la diferencia entre puntuaciones iniciales y finales en la prueba estandarizada. Por su parte, como variable independiente se utilizaron las pautas docentes de mediación puestas en práctica por los(as) profesores(as) participantes en el estudio (tipología y cantidad), ejerciendo control sobre las puntuaciones iniciales en lectura.

\section{Resultados}

\section{Distribución de los patrones de mediación}

El primer paso en el análisis de datos tiene como propósito establecer la presencia de los patrones de mediación en las sesiones observadas e identificar su frecuencia de aparición durante estas sesiones. La tabla 2 presenta los estadísticos descriptivos en relación a las observaciones realizadas en los 21 grupos-clase, divididas de acuerdo al ciclo educativo al que pertenecen (primer ciclo, de $1^{\circ}$ a $4^{\circ}$ curso, y segundo ciclo, de $5^{\circ}$ a $8^{\circ}$ ). La columna de Frecuencia presenta la cuantificación realizada en el análisis de las sesiones, estableciendo la cantidad de veces que se registra la presencia de las pautas de mediación en el grupo. La última columna presenta la Frecuencia media de las pautas de mediación codificadas, establecida en función del tiempo de observación.

Las sesiones analizadas consideraron actividades regulares de trabajo de la lectura, excluyendo aquellas sesiones en las que se realizaran pruebas de evaluación generales. También se consideró como criterio de inclusión un mínimo de dos sesiones para evitar el sesgo de una grabación aislada. La tabla muestra una relación directa entre número de sesiones, cantidad de minutos y frecuencia de pautas de mediación observadas, es decir, a mayor número de sesiones y minutos, mayor el número de frecuencia de los patrones observados. Con el propósito de reducir este sesgo se calculó la media de patrones de mediación que permite evaluar la presencia ponderada de las pautas. 
Tabla 2. Número y duración del total de sesiones de los diferentes grupos expresada en minutos, frecuencia de aparición de los patrones de mediación y promedio en función del total de minutos.

\begin{tabular}{|c|c|c|c|c|c|c|}
\hline Ciclo & Grupo & Curso & Sesiones & Tiempo & Frecuencia & $M$ Frecuencia \\
\hline \multirow{10}{*}{ Primer ciclo } & 1 & $3^{\circ}$ & 5 & 284.19 & 1560 & 5.49 \\
\hline & 2 & $3^{\circ}$ & 4 & 303.58 & 1355 & 4.46 \\
\hline & 3 & $3^{\circ}$ & 4 & 291.41 & 1248 & 4.28 \\
\hline & 4 & $3^{\circ}$ & 5 & 294.54 & 1476 & 5.01 \\
\hline & 5 & $3^{\circ}$ & 3 & 225.37 & 1074 & 4.77 \\
\hline & 6 & $4^{\circ}$ & 2 & 166.03 & 604 & 3.64 \\
\hline & 7 & $4^{\circ}$ & 2 & 165.86 & 785 & 4.73 \\
\hline & 8 & $4^{\circ}$ & 5 & 409.44 & 2147 & 5.24 \\
\hline & 9 & $4^{\circ}$ & 5 & 315.90 & 866 & 2.74 \\
\hline & 10 & $4^{\circ}$ & 4 & 313.13 & 448 & 1.43 \\
\hline \multirow{11}{*}{ Segundo ciclo } & 11 & $5^{\circ}$ & 4 & 322.16 & 1132 & 3.51 \\
\hline & 12 & $5^{\circ}$ & 4 & 253.69 & 994 & 3.92 \\
\hline & 13 & $5^{\circ}$ & 3 & 235.39 & 585 & 2.49 \\
\hline & 14 & $6^{\circ}$ & 5 & 354.10 & 662 & 1.87 \\
\hline & 15 & $6^{\circ}$ & 5 & 415.87 & 1331 & 3.20 \\
\hline & 16 & $6^{\circ}$ & 4 & 301.29 & 354 & 1.17 \\
\hline & 17 & $7^{\circ}$ & 4 & 319.57 & 894 & 2.80 \\
\hline & 18 & $7^{\circ}$ & 2 & 89.22 & 292 & 3.27 \\
\hline & 19 & $8^{\circ}$ & 3 & 199.88 & 595 & 2.98 \\
\hline & 20 & $8^{\circ}$ & 5 & 386.11 & 1491 & 3.86 \\
\hline & 21 & $8^{\circ}$ & 4 & 266.30 & 1080 & 4.06 \\
\hline Media & --- & --- & 3.90 & 281.57 & 998.71 & 3.57 \\
\hline$D T$ & --- & --- & 1.04 & 81.76 & 465.26 & 1.21 \\
\hline
\end{tabular}

Nota: $M$ Frecuencia $=$ Frecuencia media de patrones de mediación en función del tiempo de observación.

A partir de estos datos, un primer aspecto a observar es que existe una variación importante de la frecuencia media de pautas de mediación entre los distintos grupos-clase, que varía entre un promedio de 1.17 a 5.49. Estos promedios dan cuenta de una mayor o menor intensidad en el uso de las pautas de mediación por parte de los docentes y, por tanto, de una mediación más o menos enriquecida. El análisis de varianza nos permite confirmar que los grupos difieren con respecto a la frecuencia media de aparición de las pautas de mediación $\left(F_{(20,797)}=6.73 ; p<.001\right)$. Por su parte, en relación al análisis de las diferencias en función de los ciclos educativos, la frecuencia media de los grupos pertenecientes al primer ciclo, $M=4.18$ (1.26) es significativamente mayor que la de los grupos pertenecientes al segundo ciclo, $M=$ $3.01(0.89): t_{(2,19)}=2.47, p<.05$, asumiendo en el contraste la igualdad de varianzas. Es decir, los docentes que se desempeñan en grupos de estudiantes de primer ciclo utilizaron en mayor medida las pautas de mediación que los docentes que se desempeñan con estudiantes que asisten a segundo ciclo.

\section{Resultados observados sobre el progreso en lectura en los diferentes grupos}

Considerando que el objetivo central del artículo consiste en analizar la posible relación entre el progreso obtenido en lectura y las pautas de mediación utilizadas, se procedió a evaluar tanto al inicio del proceso como al cierre el rendimiento lector a través de la prueba CL-PT. La Tabla 3 presenta los puntajes promedio obtenidos por cada grupo en esta prueba en las aplicaciones inicial y final. Para verificar las diferencias entre ambas mediciones y evaluar diferencias estadísticamente significativas se utilizó la prueba $t$ para muestras relacionadas. Se incluyen así mismo el número de estudiantes, la edad promedio y la frecuencia promedio de las pautas de mediación utilizadas.

Tabla 3. Contraste (postest - pretest) entre las puntuaciones inicial y final en la prueba de lectura CL-PT.

\begin{tabular}{|c|c|c|c|c|c|c|c|c|}
\hline Ciclo & Grupo & $\mathrm{N}$ & Edad & CL-PT pre & CL-PT post & Mejora & $t(p)$ & $M$. Frec. \\
\hline \multirow{11}{*}{ Primer ciclo } & $1\left(3^{\circ}\right)$ & 26 & $8.27(1.04)$ & $26.84(15.30)$ & $42.48(10.96)$ & 15.64 & $5.20 * * *$ & 5.49 \\
\hline & $2\left(3^{\circ}\right)$ & 30 & $8.00(0.73)$ & 33.47 (15.97) & $53.68(13.59)$ & 20.22 & $7.17 * * *$ & 4.46 \\
\hline & $3\left(3^{\circ}\right)$ & 10 & $8.15(0.69)$ & $23.70(17.16)$ & $42.40(17.34)$ & 18.70 & $3.28^{* *}$ & 4.28 \\
\hline & $4\left(3^{\circ}\right)$ & 28 & $8.31(0.79)$ & $29.34(18.75)$ & $47.70(16.16)$ & 18.36 & $6.18^{* * *}$ & 5.01 \\
\hline & $5\left(3^{\circ}\right)$ & 21 & $8.06(0.66)$ & $35.83(14.07)$ & $49.64(10.38)$ & 13.81 & $5.31 * * *$ & 4.77 \\
\hline & $6\left(4^{\circ}\right)$ & 31 & $9.05(0.56)$ & $44.65(15.55)$ & $49.94(13.28)$ & 5.29 & $2.14^{*}$ & 3.64 \\
\hline & $7\left(4^{\circ}\right)$ & 30 & $9.1(0.62)$ & $38.08(26.80)$ & $47.78(11.45)$ & 9.70 & $2.11 *$ & 4.73 \\
\hline & $8\left(4^{\circ}\right)$ & 16 & $9.22(0.65)$ & $51.13(21.77)$ & $41.31(15.91)$ & -9.81 & $3.00 * *$ & 5.24 \\
\hline & $9\left(4^{\circ}\right)$ & 31 & $9.11(0.52)$ & $53.21(14.22)$ & $47.06(15.79)$ & -6.15 & $2.72 *$ & 2.74 \\
\hline & $10\left(4^{\circ}\right)$ & 29 & $9.17(0.59)$ & 40.31 (17.99) & $48.38(12.68)$ & 8.07 & $3.35^{* *}$ & 1.43 \\
\hline & & 252 & $8.68(0.84)$ & $38.36(19.91)$ & $47.67(13.86)$ & 9.31 & $8.08^{* * *}$ & 4.18 \\
\hline \multirow{12}{*}{ Segundo ciclo } & $11\left(5^{\circ}\right)$ & 23 & $10.00(0.92)$ & $31.61(17.47)$ & $53.78(23.91)$ & 22.17 & $5.37 * * *$ & 3.51 \\
\hline & $12\left(5^{\circ}\right)$ & 34 & $10.23(0.80)$ & $42.03(22.30)$ & $53.00(24.42)$ & 10.97 & $3.07 * *$ & 3.92 \\
\hline & $13\left(5^{\circ}\right)$ & 31 & $10.35(0.86)$ & $39.21(15.20)$ & $57.61(21.86)$ & 18.40 & $6.54^{* * *}$ & 2.49 \\
\hline & $14\left(6^{\circ}\right)$ & 39 & $11.05(0.82)$ & $47.14(15.74)$ & 49.09 (11.92) & 1.95 & 1.03 (ns) & 1.87 \\
\hline & $15\left(6^{\circ}\right)$ & 19 & $11.29(0.72)$ & $42.47(11.70)$ & $50.42(11.44)$ & 7.95 & $2.77^{*}$ & 3.20 \\
\hline & $16\left(6^{\circ}\right)$ & 24 & $11.09(0.51)$ & $43.96(11.78)$ & $48.32(15.68)$ & 4.36 & $1.75(\mathrm{~ns})$ & 1.17 \\
\hline & $17\left(7^{\circ}\right)$ & 41 & $12.26(0.90)$ & $36.91(16.05)$ & $31.44(12.98)$ & -5.47 & $2.39 *$ & 2.80 \\
\hline & $18\left(7^{\circ}\right)$ & 29 & $12.50(0.85)$ & $28.40(14.20)$ & $35.14(15.66)$ & 6.74 & $3.63^{* *}$ & 3.27 \\
\hline & $19\left(8^{\circ}\right)$ & 30 & $13.39(0.90)$ & $26.11(13.68)$ & $40.93(38.80)$ & 14.82 & 1.93 (ns) & 2.98 \\
\hline & $20\left(8^{\circ}\right)$ & 21 & $13.62(1.02)$ & $26.16(10.61)$ & $43.72(26.28)$ & 17.56 & $3.35^{* *}$ & 3.86 \\
\hline & $21\left(8^{\circ}\right)$ & 21 & $12.79(3.44)$ & $32.10(11.50)$ & $52.14(20.59)$ & 20.05 & $6.07 * * *$ & 4.06 \\
\hline & & 312 & $11.66(1.76)$ & $36.63(16.75)$ & $46.21(22.76)$ & 9.58 & $7.90 * * *$ & 3.01 \\
\hline Total & & 564 & $10.37(2.06)$ & $37.40(18.23)$ & $46.86(19.31)$ & 9.46 & $11.19 * * *$ & 3.57 \\
\hline
\end{tabular}

Nota: ${ }^{*} p<.05 ; * * p<.01 ;{ }^{* * *} p<.001 ; M$. Frec. = Frecuencia media de patrones de mediación/minuto. 
Los resultados muestran ganancias promedio estadísticamente significativas en 15 grupos; otros tres grupos, 14, 16 y 19 , presentan mejoras en sus puntuaciones promedio, aunque éstas no son significativas. Por su parte, los grupos 8,9 y 17 muestran pérdidas que llegan a ser significativas. En razón de los resultados observados, se comparó el progreso en el rendimiento lector por ciclo educativo. Considerando cada ciclo como un solo grupo, se obtienen diferencias significativas en ambos ciclos.

En la Tabla 4 se presentan las frecuencias promedio de las pautas de mediación agrupadas de acuerdo al sistema de categorías establecido (Navarro et al., 2014). La primera dimensión, (a) mediación de aspectos generales, contempla pa- trones de mediación de tipo general, no ligados directamente a ningún proceso lector; la segunda, (b) mediación del conocimiento metacognitivo y uso de estrategias de lectura, incorpora los aspectos metacognitivos involucrados en el aprendizaje de la lectura y también aquellos aspectos relacionados con el uso de estrategias de integración textual e integración del texto con los conocimientos y experiencias previas. La tercera dimensión, (c) mediación de procesos de ajuste personal-social, contiene elementos de carácter personal, asociados a la motivación e interés por la lectura, y grupal, vinculados a las dinámicas de apoyo y participación entre los estudiantes.

$\underline{\text { Tabla 4. Frecuencia media de aparición de las pautas de mediación por dimensiones y ciclo educativo. }}$

\begin{tabular}{lcccc}
\hline & $\begin{array}{c}\text { Mediación de aspectos } \\
\text { generales }\end{array}$ & \begin{tabular}{l} 
Mediación del conocimiento metacognitivo y del uso de estrategias \\
\cline { 3 - 5 }
\end{tabular} & $\begin{array}{c}\text { Conocimiento metacognitivo } \\
\text { Uso de estrategias }\end{array}$ & $\begin{array}{c}\text { Medón de procesos de ajuste } \\
\text { personal-social }\end{array}$ \\
\hline Primer Ciclo & 1.18 & 0.07 & 1.71 & 1.22 \\
Segundo Ciclo & 0.92 & 0.06 & 1.29 & 0.72 \\
\hline Total & 1.05 & 0.06 & 1.50 & 0.96 \\
\hline
\end{tabular}

En términos generales, la dimensión que tuvo una mayor frecuencia promedio fue la de mediación del conocimiento metacognitivo y uso de estrategias de lectura. Sin embargo, se observaron diferencias importantes al interior de esta dimensión entre la subdimensión asociada al conocimiento metacognitivo y la otra referida a las estrategias de lectura. Esta segunda subdimensión incluye pautas sobre estrategias de autorregulación, integración textual y activación e integración de conocimientos previos. Las pautas de mediación menos observadas en el desempeño de los docentes fueron aquellas referidas a la subdimensión sobre el conocimiento metacognitivo. A partir de los resultados analizados inicialmente, pareció relevante también observar posibles diferencias por ciclo. Los resultados mostraron que todas las dimensiones fueron observadas con mayor frecuencia en docentes del primer ci- clo, y en especial la presencia de las pautas correspondientes a la dimensión Mediación del uso de estrategias de lectura.

\section{Progreso lector y utilización de pautas de mediación en la lectura}

Con el propósito de evaluar la relación entre el progreso en el rendimiento lector observado en los grupos y los patrones de mediación utilizados por los docentes en aula, se efectuaron en primer lugar análisis de correlación entre las diferencias postest - pretest y el uso de los patrones de mediación. Los resultados se observan en la tabla 5. Hemos incluido en estos análisis las variables sexo, edad y número de estudiantes por curso.

Tabla 5. Análisis de correlación entre las distintas variables contempladas en el estudio.

\begin{tabular}{|c|c|c|c|c|c|c|}
\hline & Estudiantes por curso & Edad & Sexo & CL-PT Postest & Mejora & Frecuencia \\
\hline Estudiantes por curso & --- & & & & & \\
\hline Edad & $.11^{* *}$ & --- & & & & \\
\hline Sexo & .02 & $.14 * * *$ & --- & & & \\
\hline CL-PT Postest & -.01 & $-.19 * * *$ & $-.13 * *$ & --- & & \\
\hline Mejora & $-.09 *$ & -.05 & -.05 & $.57 * * *$ & --- & \\
\hline Frecuencia & $-.30 * * *$ & $-.27 * * *$ & -.05 & -.04 & -.06 & --- \\
\hline$M$ Frecuencia & $-.49 * * *$ & $-.32 * * *$ & -.05 & -.03 & $.14^{* *}$ & $.51 * * *$ \\
\hline
\end{tabular}

Nota: ${ }^{*} p<.05 ; * * p<.01 ;{ }^{* *} p<.001$ (Bilateral); $M$ Frecuencia = Frecuencia media de patrones de mediación en función del tiempo de observación.

Los análisis de correlación de Pearson mostraron un índice significativo, aunque débil, entre el promedio de frecuencia y las puntuaciones de mejora en comprensión lectora. Así mismo, el número de estudiantes por curso y la edad correlacionaron negativamente con la frecuencia media de anotaciones. Por su parte, los análisis efectuados en función del ciclo educativo no ofrecieron en lo esencial cambios relevantes con respecto a los resultados globales. Tan solo destacar que en ambos ciclos se incrementó el nivel de correlación entre la frecuencia promedio de patrones de mediación utilizados y las mejoras observadas, llegando a ser de $r=.18$ para el primer ciclo, y de $r=.17$ para el segundo. Así mismo, en el primer ciclo se observó una correlación negativa y significativa entre el sexo y la frecuencia promedio de patrones, lo que indicaría una relación inversa entre el número de niños varones y la frecuencia media de patrones de mediación.

Con el propósito de dilucidar en qué medida el uso de los patrones de mediación observados explicaba de forma general el progreso en rendimiento lector, se realizaron análisis de regresión utilizando las puntuaciones de mejora como 
variable dependiente y la frecuencia promedio de los patrones de mediación con variable independiente. La Tabla 6 presenta los resultados de estos análisis. Considerando los 21 grupos y el total de los patrones de mediación en conjunto, es posible observar que la frecuencia media de las pautas de mediación explicaba un $2 \%$ de la varianza en las puntuaciones de mejora en la comprensión, medida con la prueba CLPT. En el primer ciclo, esta incidencia en la varianza de la mejora llegaba a ser del 3.2\%, mientras que en el segundo ciclo fue del $2.7 \%$. En cuanto a las variables Estudiantes por curso y Edad, solo la primera logró entrar en la ecuación de regresión, si bien el poder explicativo fue también escaso. Por su parte, los valores del estadístico Durbin-Watson fueron adecuados, situándose entre 1.79 para el modelo correspondiente a la frecuencia media de los patrones de mediación, y 1.67 para el modelo correspondiente a la variable Edad. En síntesis, consideradas en conjunto, las pautas de mediación observadas, si bien inciden en el rendimiento lector, presentan una limitada capacidad explicativa sobre el progreso de los estudiantes.

Tabla 6. Análisis de regresión en relación a las puntuaciones de mejora sobre el uso de los patrones de mediación docentes en el aula.

\begin{tabular}{|c|c|c|c|c|c|}
\hline \multirow{2}{*}{$N=564$} & \multicolumn{5}{|c|}{ Mejora } \\
\hline & Beta & $\mathrm{R}^{2}$ ajustada & $\Delta R^{2}$ & Residual & $F(p)$ \\
\hline$\overline{M \text { Frecuencia }}$ & .14 & .02 & .02 & 19.89 & $11.30^{* *}$ \\
\hline Estudiantes por curso & -.09 & .01 & .01 & 20.02 & $4.57 *$ \\
\hline Edad & -.05 & .00 & .00 & 20.22 & 1.31 (n.s) \\
\hline
\end{tabular}

Nos disponemos a continuación a identificar aquellos patrones de mediación más utilizados en los distintos grupos, así como la relación entre los patrones de mediación utilizados y las mejoras obtenidas. En la Tabla 7 se presenta la frecuencia media de aparición de los patrones para cada uno de los grupos-clase observados. En este sentido, es posible identificar los patrones de mediación más utilizados en aquellos grupos que obtuvieron diferencias favorables en el postest. Los patrones de mediación: PM_3, Establecimiento de apoyo en la comprensión, secuenciación o realización de la actividad (0.73); PM_16, Mediación del uso de estrategias de integración textual (0.91); y PM_18, Mediación de la motivación, la actitud y el in- terés con relación a la lectura (0.87), fueron los que presentaron mayor frecuencia promedio en la práctica totalidad de los grupos, tanto aquellos que obtuvieron ganancias postest, como aquellos que obtuvieron ganancias no significativas o incluso pérdidas. Cabe destacar asimismo que en los tres casos los patrones de mediación identificados, presentaron mayor frecuencia promedio en los grupos del primer ciclo, llegando a ser esta diferencia significativa estadísticamente con respecto al segundo ciclo para el PM_3 $(t(2,19)=2.87, p$ $=.01)$, y el PM_18 $\left(t_{(2,19)}=2.50, p<.05\right)$. En ambos casos se asumió la igualdad de varianzas.

Tabla 7. Patrones de mediación más utilizados en aquellos grupos que obtuvieron mejoras entre las fases inicial y final del estudio, y relación con las mejoras.

\begin{tabular}{|c|c|c|c|c|c|c|c|c|c|c|c|c|c|c|c|c|c|c|c|c|c|c|c|}
\hline \multirow{2}{*}{$\begin{array}{c}\text { Patrones } \\
\text { de Mediación }\end{array}$} & \multicolumn{23}{|c|}{ Frecuencia media de los patrones de mediación en función del tiempo de observación para cada grupo-clase } \\
\hline & 1 & 2 & 3 & 4 & 5 & 6 & 7 & 8 & 9 & 10 & 11 & 12 & 13 & 14 & 15 & 16 & 17 & 18 & 19 & 20 & 21 & & \\
\hline PM_1 & 0.23 & 0.31 & 0.04 & 0.13 & 0.42 & 0.11 & 0.09 & 0.36 & 0.13 & 0.05 & 0.14 & 0.26 & 0.08 & 0.06 & 0.34 & 0.05 & 0.13 & 0.04 & 0.07 & 0.09 & 0.15 & 0.16 & -.06 \\
\hline PM_2 & 0.00 & 0.01 & 0.01 & 0.00 & 0.00 & 0.00 & 0.00 & 0.05 & 0.00 & 00 & 0.00 & 0.00 & 0.02 & 0.00 & 0.00 & 0.00 & 0.00 & 0.00 & 0.00 & 0.00 & 0.00 & 0.00 & -.30 \\
\hline PM_3 & 1.32 & 1.08 & 1.04 & 0.94 & 0.90 & 0.66 & 0.87 & 0.88 & 0.65 & 43 & 0.61 & 0.86 & 0.40 & 0.69 & 0.72 & 0.24 & 0.84 & 0.29 & 0.62 & 0.64 & +0.60 & 73 & 15 \\
\hline PM_4 & 0.17 & 0.02 & 0.04 & 0.11 & 0.08 & 0.07 & 0.04 & 0.04 & 0.07 & 0.00 & 17 & 0.15 & 0.02 & 0.01 & 0.02 & 0.00 & 0.09 & 58 & 0.04 & 0.41 & 0.36 & 12 & .21 \\
\hline M_P5 & 0.04 & 0.00 & 00 & 0.03 & 0.02 & 0.00 & & 0.01 & 0.00 & 00 & & 0.00 & .00 & 0.00 & 0.01 & 0.00 & 00 & 00 & 0.00 & 0.00 & 0.00 & 01 & .21 \\
\hline & 0.01 & 0.02 & 0. & 0.03 & 0.01 & 0. & & & 0.02 & & & 0.01 & 00 & 01 & 0.02 & .00 & 01 & 00 & 0.02 & 0.00 & 0.00 & 01 & -.33 \\
\hline I_P7 & 0.00 & 0.04 & 0 & 0.01 & 0.01 & 0.02 & & 0. & 0.01 & 0 & & 0.01 & .00 & 00 & 0.01 & 0.00 & 01 & 00 & 0.01 & 0.00 & 0.00 & 01 & -.11 \\
\hline & 0.00 & .05 & & 0.09 & 0.00 & 0. & & & 0.02 & & & 0.02 & .01 .01 & 0.01 & 0.02 & 0.01 & 0.06 & 00 & 0.13 & 0.00 & 0.02 & 03 & .06 \\
\hline & 0.00 & 0.00 & 0.00 & 0.01 & 0.03 & 0. & & 0.00 & 0.02 & & & 0.02 & 0.02 & 0.00 & 0.03 & 0.02 & .06 & 00 & 0.01 & 0.03 & 0.05 & .02 & . 20 \\
\hline & 0.00 & 0.01 & 0.08 & 0.00 & 0.00 & 0. & 0.02 & 0.00 & 0.01 & 0 & 0.00 & 0.00 & 0.00 & 0.00 & 0.01 & 0.00 & 0.01 & 0.00 & 0.01 & 0.00 & 0.00 & .01 & .13 \\
\hline & 0.00 & 0.00 & 0.00 & 0.00 & 0.00 & 0.00 & 0.00 & 0.00 & 0.00 & 0 & 0.00 & 0.00 & 0.00 & 0.00 & 0.00 & 0.00 & 0.00 & 0.00 & 0.00 & 0.00 & 0.00 & .00 & .00 \\
\hline & 0.05 & 0.04 & 0. & 0.05 & 0.02 & 0.05 & 0.04 & 0.06 & 0.05 & 0 & 0.06 & 0.02 & 0.02 & 0.03 & 0.00 & 0.00 & 0.03 & 0.01 & 0.02 & 0.01 & 0.03 & .03 & -.04 \\
\hline & 0.45 & 0.24 & 0.20 & 0.39 & 0.41 & 0.37 & 0.46 & 0.36 & 0.21 & 0 & 0.29 & 0.24 & 0.10 & 0.24 & 0.29 & 0.04 & 0.18 & 0.15 & 0.22 & 0.07 & 0.18 & .25 & -.01 \\
\hline & 0.04 & 0.30 & 0.46 & 0.13 & 0.07 & 0.27 & 0.24 & 0.33 & 0.21 & 0 & 0.02 & 0.15 & 0.02 & 0.02 & 0.23 & 0.07 & 0.07 & 0.07 & 0.33 & 0.06 & 0.05 & .15 & -.11 \\
\hline 15 & 0.01 & 0.00 & 0.00 & 0.01 & 0.00 & 0.01 & 0.00 & 0.00 & 0.00 & 0.00 & 0.00 & 0.00 & 0.00 & 0.00 & 0.00 & 0.00 & 0.00 & 0.00 & 0.03 & 0.00 & 0.00 & .00 & .17 \\
\hline 16 & 1.16 & 0.88 & 0.86 & 1.38 & 0.92 & 1.13 & 1.27 & 1.58 & 0.60 & 0.27 & 1.26 & 1.03 & 1.10 & 0.46 & 0.76 & 0.38 & 0.42 & 1.02 & 0.66 & 0.99 & 1.07 & 0.91 & .27 \\
\hline 17 & 0.34 & 0.12 & 0.22 & 0.13 & 0.03 & 0.11 & 0.13 & 0.11 & 0.09 & 0.02 & 0.32 & 0.15 & 0.31 & 0.04 & 0.02 & 0.02 & 0.16 & 0.09 & 0.24 & 0.35 & 0.25 & 0.15 & $.55^{* *}$ \\
\hline-18 & 1.40 & 1.21 & 1.12 & 1.48 & 1.26 & 0.74 & 1.28 & 1.25 & 0.61 & 0.34 & 0.48 & 0.89 & 0.37 & 0.30 & 0.54 & 0.34 & 0.60 & 1.02 & 0.59 & 1.15 & 1.23 & 0.87 & .28 \\
\hline PM_19 & 0.26 & 0.15 & 0.15 & 0.11 & 0.59 & 0.06 & 0.07 & 0.12 & 0.03 & 0.03 & 0.03 & 0.09 & 0.02 & 0.02 & 0.16 & 0.01 & 0.13 & 0.00 & 0.04 & 0.04 & 0.07 & 0.10 & .13 \\
\hline
\end{tabular}

En relación a los contrastes entre la frecuencia media de los patrones de mediación y la mejora obtenida, el PM_17,
Mediación del uso de estrategias para la activación de conocimientos previos y la integración de conocimientos y experiencias con nuevos con- 
tenidos, obtuvo un nivel de correlación significativa con la mejora $(r=.55, p<.01)$. Este patrón de mediación explicaba el $26 \%$ de la varianza en la mejora, resultando estadísticamente significativo $\left(R^{2 a j u s t a d o}=.26, F_{(1,19)}=8.19, p=.01\right) . \mathrm{El}$ PM_2, Ampliación de la actividad mediante la realización de tareas relacionadas que surgen del profesor o del propio alumnado, obtuvo un nivel de correlación de $r=-.42(p<.05)$ con la mejora cuando se tomó en cuenta la suma de anotaciones de los patrones y no el promedio de frecuencia en función del tiempo observado. Así mismo, el PM_6, Repetición de una actividad para poder poner en práctica las estrategias trabajadas y para poder evaluar su incorporación a la ejecución de la actividad por parte del alumno, también obtuvo un nivel de correlación negativa y significativa $(r=-.44, \mathrm{p}<.05)$ con la mejora, mostrando, al igual que en el caso anterior, que este patrón se habría observado especialmente ante la presencia de mayores dificultades en los grupos. En este caso, este patrón de mediación explicaba el $15 \%$ de la varianza en la mejora, resultando estadísticamente significativo $\left(R^{2 a j u s t a d o}=.15, F_{(1,19)}=4.50, p<.05\right)$. El PM_4, Recapitulación conjunta del trabajo realizado anteriormente con el objetivo de retomarlo, obtuvo un nivel de correlación marginalmente significativo $(r=.31, p=.08)$ con la mejora cuando se tomó en cuenta la frecuencia de los patrones, explicando un $10 \%$ de la varianza en las puntuaciones de mejora.

Finalmente, en relación a las diferencias por ciclos, los análisis mostraron para el primer ciclo que el patrón PM_3 obtenía un nivel de relación marginalmente significativo con la mejora $(r=.52, p<.06)$, así como el PM_2 $(r=-.52, p=$ .06) y PM_6 $(r=-.45, p=.09)$. Por su parte, para el segundo ciclo, el patrón PM_16 mostraba una relación muy significativa con la mejora $\left(r=.82, p=.001 ; R^{2 a j u s t a d o}=.63, F_{(1,9)}=\right.$ $18.34, p=.002$ ), explicando el $63 \%$ de su varianza. Así mismo, el PM_17 mostraba especialmente su poder explicativo en el segundo ciclo $\left(r=.75, p=.004 ; R^{2 \text { ajustado }}=.52, F_{(1,9)}=\right.$ $11.61, p=.008)$.

\section{Discusión}

El presente estudio pretendía identificar patrones docentes de mediación en actividades específicas de lectura que actuaran como factores decisivos en las mejoras obtenidas en el rendimiento lector de estudiantes de educación básica en contextos de desventaja sociocultural. En términos más específicos, la hipótesis de trabajo sostenía que el mayor progreso en rendimiento lector estaría relacionado significativamente con la implementación de patrones de mediación de carácter metacognitivo y autorregulatorio, así como de aquellos más relacionados con los procesos de ajuste personalsocial.

Los resultados muestran de forma general que los grupos que obtuvieron mayores promedios de frecuencia en el uso de los patrones de mediación, obtuvieron mayores ganancias. La relación que se establece es significativa, aunque débil cuando se analiza globalmente. A tenor de los resultados, los cambios observados en rendimiento lector se explican en un pequeño porcentaje por la utilización de los patrones de me- diación observados. Los resultados muestran así mismo una implementación limitada, tanto en relación a la variedad de patrones utilizados por los docentes, como a la intensidad en el uso. En este sentido, los más utilizados de entre los diecinueve que fueron recogidos inicialmente en el sistema de categorías fueron: el patrón PM_16, Mediación del uso de estrategias de integración textual; el PM_3, Establecimiento de apoyo en la comprensión, secuenciación o realización de la actividad; y el PM_18, Mediación de la motivación, la actitud y el interés con relación a la lectura. Estos tres obtuvieron una mayor frecuencia en 20 de los 21 grupos-clase observados. Debemos señalar que estos resultados son coherentes con la relevancia otorgada a estos procesos en distintos trabajos mencionados previamente, bien porque son parte integrante de la propia conceptualización de los procesos de comprensión (Kintsch, 1988; Rueda, 2013; Sánchez, 2010), o bien porque han sido utilizados en estudios de carácter empírico en los que se ha evaluado su impacto en la competencia lectora (Beker et al., 2016; Elbro \& Buch-Iversen, 2013; Thiede et al., 2009; van Driel et al., 2018).

Los análisis dirigidos a establecer específicamente la incidencia de cada uno de los patrones de mediación en la explicación del cambio en el rendimiento lector, mostraron que el patrón PM_17, Mediación del uso de estrategias para la activación de conocimientos previos y la integración de conocimientos y experiencias con nuevos contenidos, explicaba el $26 \%$ de la varianza en la mejora, resultando estadísticamente significativo, y que el PM_4, Recapitulación conjunta del trabajo realizado anteriormente con el objetivo de retomarlo, lograba explicar un $10 \%$ de la varianza, alcanzando en este caso un nivel de significatividad marginal. En este sentido, con respecto al patrón PM_17, los resultados son consistentes con las propuestas de diversos autores (Kintsch, 1988; Sánchez, 2010; León et al., 2012), así como con algunos estudios en los que específicamente se analizan estos procesos (Beker et al., 2016; Elbro \& BuchIversen, 2013). En relación al patron PM_4, debemos señalar que las pautas concretas de mediación que lo integran, hacen referencia a aspectos muy próximos a la activación de conocimientos previos: Reconstruiry revisar conjuntamente el trabajo realizado en sesiones anteriores, con el objetivo de explicitar los logros conseguidos y plantear nuevas metas, y Preguntar por actividades realizadas anteriormente. Es posible que esta sintonía explique los resultados observados a nivel estadístico.

Por otra parte, dos patrones de mediación correlacionaron negativa y significativamente con la mejora: el patrón PM_2 y el PM_6. El primero de ellos hace referencia a la ampliación de la actividad mediante la realización de tareas relacionadas que surgen del profesor o del propio alumnado, lo que podría indicar que su puesta en práctica habría tenido lugar ante una mayor necesidad de profundización y apoyo, lo que se daría precisamente en aquellos grupos con mayores dificultades. En este sentido, los grupos con mayores ganancias no habrían requerido de este tipo de estrategias. Por su parte, el PM_6, hace referencia a la repetición de una actividad para poder poner en práctica las estrategias trabajadas y para poder evaluar su incorporación a la ejecución de la actividad por parte del alumno, lo que 
podría indicar, al igual que en el caso anterior, que su aplicación habría tenido lugar especialmente en aquellos casos en los que fue necesaria la repetición de actividades, revelando la presencia de mayores dificultades. En total fueron 7 las correlaciones negativas entre la frecuencia media de los patrones de mediación y la mejora en rendimiento lector, si bien solo dos de ellas mostraron significatividad. Este resultado es coherente con el obtenido en un estudio previo ( $\mathrm{Na}$ varro et al., 2014), en el que la observación de estos mismos patrones de mediación se relacionó positivamente con la necesidad de mediación valorada por los docentes (llegando a ser significativa esta relación para el patrón PM_6), y, en menor medida, con las mejoras observadas. Es decir, la presencia de algunos patrones de mediación tendría lugar, en mayor medida, como respuesta a la presencia de dificultades en el proceso, que como estrategias proactivas de enriquecimiento.

Un aspecto no considerado inicialmente en el estudio fue el análisis de las diferencias observadas entre los ciclos educativos. Las observaciones efectuadas revelaron la presencia de diferencias significativas en la implementación de pautas de mediación en función del ciclo, encontrándose una mayor frecuencia promedio en el primero. Los patrones de mediación más utilizados en el estudio (PM_3, PM_16 y PM_18) también lo fueron en mayor medida en el primer ciclo, si bien estas diferencias no se reflejaron en un mayor progreso en el rendimiento lector de estos estudiantes. En relación a este dato, cabe señalar que el profesorado de cursos mayores nos comunicó en un Seminario posterior a la finalización del estudio, que esperaba de su alumnado que accediera a la comprensión sin necesidad de ofrecer determinadas ayudas de carácter general o motivacional, que consideraban en ocasiones más propias de estudiantes de menor edad. Por otra parte, los análisis en función del ciclo nos permitieron observar también diferencias relevantes en la relación existente entre patrones de mediación y progreso lector. En este sentido, los patrones PM_2, PM_3 y PM_6 resultaron especialmente significativos para el primer ciclo, mientras que para el segundo los patrones PM_16 y PM_17, es decir, aquellos que hacen referencia a la mediación de estrategias de integración textual, y de estrategias de integración del texto con los conocimientos previos, explicaban significativamente el cambio en rendimiento lector. De este modo, aquellos patrones de mediación dirigidos a las estrategias de lectura de mayor complejidad, resultaron especialmente claves en la explicación de las mejoras obtenidas por los estudiantes de cursos mayores. Estos resultados están en consonancia con el trabajo fundamental de Kintsch (1988), así como con los datos obtenidos en estudios más recientes en los que se pone de manifiesto la relevancia de estos procesos en la comprensión lectora (Beker et al., 2016; Elbro \& Buch-Iversen, 2013; León et al., 2012).

Debemos destacar así mismo que algunos de los patrones de mediación contemplados inicialmente en el sistema de categorías, no fueron observados durante las grabaciones analizadas y, como consecuencia, no fueron codificados (PM_11, Conciencia de las propias dificultades relacionadas con la lec- tura; y PM_15, Mediación del uso de estrategias de transferencias y generalización). Este resultado podría ser relevante por cuanto se aleja de algunas de las conclusiones establecidas a raíz de la evaluación de algunas propuestas de intervención y dispositivos de evaluación dinámica, que han mostrado precisamente mayor eficacia cuando se mediaba la puesta en práctica de habilidades metacognitivas y de estrategias de transferencia (Aguilera \& Mora, 2004; Beckmann et al., 2009; Carlson \& Wiedl, 2000; Resing et al., 2009; Resing \& Elliott, 2011; Souvignier \& Mokhlesgerami, 2006; Thiede et al., 2009).

Una interpretación del relativo bajo impacto de las pautas de mediación en el progreso lector, se basaría precisamente en la escasa aparición de pautas relacionadas con el conocimiento metacognitivo y las estrategias de autorregulación. Tal como se señalaba en la hipótesis de trabajo, se esperaba que un mayor progreso en el rendimiento lector de los estudiantes estuviera relacionado significativamente con este tipo de pautas. En este sentido, nuestros resultados parecen estar en sintonía con el estudio mencionado relativo a los patrones instruccionales (Preiss, 2009). Este estudio revelaba el predominio de interacciones pedagógicas caracterizadas por el control externo por parte del docente, y el seguimiento del alumno en la adquisición de información. Cabría pensar que, en un escenario de mayor variedad de pautas de mediación, y con un énfasis mayor en pautas asociadas a procesos metacognitivos y de autorregulación, el efecto global sobre el progreso lector habría sido quizás más alto. Por otra parte, sí se ha puesto de manifiesto la relevancia específica de la mediación del uso de estrategias relacionadas con la activación e integración de conocimientos y experiencias previas con el nuevo contenido de aprendizaje. Se trataría de procesos de alto nivel y especial relevancia en la explicación de la comprensión de textos (Graesser et al., 1994; Hacker, 1998; Sánchez, 2010).

\section{Limitaciones del presente estudio y nuevas vías de análisis}

Como hemos descrito, el estudio aborda el efecto de los patrones de mediación en los resultados de aprendizaje, y trata de identificar algunas claves en torno a la mediación más efectiva en estudiantes de contextos desfavorecidos. Es necesario sin embargo abordar las limitaciones del presente estudio con el objetivo de avanzar en los objetivos planteados y llegar a conclusiones más definitivas. En primer lugar, si bien los programas de enriquecimiento cognitivo aportan elementos de juicio para evaluar la riqueza de los procesos de mediación, no disponemos de parámetros precisos que nos permitan realizar comparaciones y evaluar en qué nivel de calidad se encontrarían los procesos de mediación observados en aula. En segundo lugar, tampoco disponemos de parámetros para evaluar el progreso en el rendimiento lector de los estudiantes en el marco temporal del año escolar. En este sentido, si bien lo esperable es que los estudiantes progresen, no podemos determinar con precisión si el progreso obser- 
vado se encuentra en un rango esperado. En este contexto, los resultados obtenidos podrían ser relevantes, no solo para identificar aquellos patrones de mediación que pueden estar en la base del progreso lector de estudiantes que se encuentran en situación de desventaja sociocultural, sino también para ir estableciendo conocimiento acumulado en relación al progreso observado, a las pautas de mediación utilizadas por el profesorado en estos contextos, así como a la respuesta a la mediación de los estudiantes. Debemos reseñar por último que en este artículo no profundizamos en las variaciones de las pautas de mediación en relación a las características individuales de los docentes, como años de experiencia laboral, grados de perfeccionamiento, género u otras variables individuales. Ni así mismo, en la variabilidad en la respuesta a la mediación como consecuencia de características individuales de los estudiantes. Futuros estudios de profundización deberán analizar el efecto de estas variables en la relación entre patrones de mediación y progreso lector.

En síntesis, el estudio presentado ha logrado identificar una serie de patrones docentes de mediación relacionados significativamente con el progreso lector de estudiantes en situación de desventaja sociocultural. En general, aquellos grupos en los que se aplicaron con más frecuencia los patrones de mediación analizados, obtuvieron mayores ganancias, aunque se observaron diferencias relevantes en función del

\section{Referencias}

Agencia de Calidad de la Educación (2016a). Evolución de las brechas socioeconómicas de rendimiento en pruebas SIMCE [Evolution of socio-economic gaps of performance in SIMCE tests]. Santiago, Chile: Ministerio de Educación.

Agencia de Calidad de la Educación (2016b). Estudios Internacionales [International Studies]. Recuperado de: https://www.agenciaeducacion.cl/estudios/estudiosinternacionales/estudios-desarrollados/

Aguilera, A., \& Mora, J. (2004). Interaction and cognitive development: Cognitive Mobilizing Patterns (CMP). Psychology in Spain, 8(1), 16-27.

Ato, M., López-García, J. J., \& Benavente, A. (2013). A classification system for research designs in psychology. Anales de Psicología, 29(3), 1038-1059. https://doi.org/10.6018/analesps.29.3.178511

Beker, K., Jolles, D., Lorch, R.F., \& van den Broek, P. (2016). Learning from texts: activation of information from previous texts during reading. Reading and Writing, 29(6), 1161-1178. https://doi.org/10.1007/s11145-016-9630-3

Beckmann, N., Beckmann, J. F., \& Elliott, J. G. (2009). Self-confidence and performance goal orientation interactively predict performance in a reasoning test with accuracy feedback. Learning and Individual Differences, 19(2), 277-282. doi: http://dx.doi.org/10.1016/j.lindif.2008.09.008

Bellei, C., Valenzuela, J.P., Vanni, X., \& Contreras, D. (2014). Lo aprendí en la escuela ¿Cómo se logran procesos de mejoramiento escolar? [I learned it at school. How are school improvement processes achieved?] Santiago, Chile: Universidad de Chile.

Boardman, A.G., Boelé, A.L., \& Klingner, J.K. (2018). Strategy Instruction Shifts Teacher and Student Interactions During Text-Based Discussions. Reading Research Quarterly, 53(2), 175-195. https://doi.org/10.1002/rrq.191

Bronfenbrenner, U. (1987). La ecología del desarrollo bumano. Barcelona, España: Paidós [The ecology of human development: Experiments by nature and designs, Cambridge, MA: Harvard University Press, 1979].

Cadiz, J., \& Martinic, S. (2007). Variables de eficacia escolar en contextos de pobreza. El caso del P900 en Chile [Variables of school effectiveness in ciclo educativo en cuanto a la relación entre patrones de mediación y progreso lector. En este sentido, el apoyo en la comprensión, secuenciación o realización de las actividades, parece haber sido clave en el progreso de los estudiantes de primer ciclo, mientras que la mediación de estrategias de integración textual, y de la activación e integración de conocimientos previos con nuevos contenidos, se habría revelado como un factor decisivo en el progreso de los estudiantes de segundo ciclo. De este modo, en función de qué patrones de mediación resultaron claves en la explicación de las mejoras observadas, es posible inferir algunas pautas que permitirían optimizar el aprendizaje y orientar la intervención.

Financiación: Este trabajo ha recibido financiación de las siguientes fuentes: Proyectos DIP 29-2014 y DIP 45-2015, Fondos internos de Apoyo a la Investigación, Dirección de Postgrado e Investigación de la Universidad Autónoma de Chile; Proyecto APIX 2-0216, Universidad Metropolitana de Ciencias de la Educación; Grupo de Investigación Educación y Procesos Psicológicos, Universidad de Zaragoza.

Agradecimientos.- Agradecemos especialmente la colaboración de los establecimientos educativos participantes en el estudio.

Conflicto de intereses: Los autores declaran que no existen conflictos de interés. contexts of poverty: The case of the P900 in Chile]. REICE - Revista Iberoamericana sobre Calidad, Eficacia y Cambio en Educación, 5(5e), 229-243.

Carlson, J. S., \& Wiedl, K. H. (2000). The validity of dynamic assessment. En C. S. Lidz \& J. G. Elliot (Eds.), Dynamic Assessment: Prevailing Models and Applications (6) (pp. 681-712). Greenwich, CT: Elsevier-JAI.

Compton, D. L., Fuchs, D., Fuchs, L., Bouton, B., Gilbert, J., Barquero, L., ... Crouch, R. C. (2010). Selecting at-risk first-grade readers for early intervention: eliminating false positives and exploring the promise of a two-stage gated screening process. Journal of Educational Psychology, 102(2), 327-340. http://dx.doi.org/10.1037/a0018448

Elbro, C., \& Buch-Iversen, I. (2013). Activation of background knowledge for inference making: Effects on reading comprehension. Scientific $\begin{array}{lll}\text { Studies } & \text { Reading, 17(6), }\end{array}$ https://doi.org/10.1080/10888438.2013.774005

Flecha, A., Garcia, R., Gomez, A., \& Latorre, A. (2009). Participation in successful schools: A communicative research study from the Includeed Project. Cultura y Educación, 21(2), 183-196.

Fernald, A., Marchman, V.A., \& Weisleder, A. (2013). SES differences in language processing skill and vocabulary are evident at 18 months. Developmental Science, 16(2), 234-248. https://doi.org/10.1111/desc.12019

Flouri, E., Midouhas, E., Joshi, H., \& Sullivan, A. (2016). Socioeconomic Disadvantage and Children's Behaviour Problems: the Role of Early Aspirations. Longitudinal and Life Course Studies, 7(2), 144-164. http://dx.doi.org/10.14301/llcs.v7i2.364

Graesser, A.C., Singer, M., \& Trabasso, T. (1994). Constructing inferences during narrative test comprehension. Psychological Review, 101(3), 371-395.

Grigorenko, E. (2009). Dynamic assessment and response to intervention. Two sides of one coin. Journal of Learning Disabilities, 42(2), 111-132. http://dx.doi.org/10.1177/0022219408326207

Hacker, D. (1998). Self-regulated comprehension during normal reading. In D. Hacher, J. Dunlosky, \& A. Graesser (Eds.), Metacognition in educational theory and practice (pp. 165-192). Hillsdale, N.J.: Lawrence Earlbaum Associates.

Hou, Y.-J. (2018). Comparing teacher- and student-focused instruction on the development of critical thinking skills and reading comprehen- 
sion. International Journal of Literacies, 25(1), 11-26. doi:10.18848/23270136/CGP/v25i01/11-26

Junta Nacional de Auxilio Escolar y Becas (JUNAEB) (2012). Informe sobre Índices de Vulnerabilidad Escolar (IVE) [school vulnerability indexes report]. Santiago, Chile: Ministerio de Educación de Chile.

Kieffer, M. J. (2012). Before and after third grade: Longitudinal evidence for the shifting role of socioeconomic status in reading growth. Reading and Writing, 25(7), 1725-1746. https://doi.org/10.1007/s11145-011-9339-2

Kintsch, W. (1988). The role of knowledge in discourse comprehension: A construction-integration model. Psychological Review, 95(2), 163-182.

León, J.A., Escudero, I., \& Olmos, R. (2012). Evaluación de la comprensión lectora (ECOMPLEC). Madrid: TEA Ediciones.

Ley de Subvención Escolar Preferencial (2008). Ley de Subvención Escolar Preferencial [preferential school grant law] (Ley SEP) $N^{\circ} 20.248$, Congreso Nacional de Chile, 2008.

Medina, A., Gajardo, A.M., \& Fundación Arauco (2010). Pruebas de comprensión lectora y producción de textos (CL-PT) [Tests of reading comprehension and text production (CL-PT)]. Santiago de Chile: Ediciones UC.

Muijs, D, (2003) La mejora y la eficacia de las escuelas en zonas desfavorecidas: resumen de resultados de investigación [The improvement and effectiveness of schools in disadvantaged areas: summary of resarch results]. Revista electrónica Iberoamericana sobre Calidad, eficacia y cambio en Educación. 1(2), 1-7.

Navarro, J.-J. \& Mora, J. (2011). Analysis of the implementation of a dynamic assessment device of processes involved in reading with learningdisabled children, Learning and Individual Differences, 21(2), 168-175. http://dx.doi.org/10.1016/j.lindif.2010.11.008

Navarro, J.-J. \& Mora, J. (2013). Validez predictiva e incremental de un dispositivo de evaluación dinámica sobre el rendimiento y el progreso en lectura. Anales de Psicología, 29, 2, 435-453. http://dx.doi.org/10.6018/analesps.29.2.135231

Navarro, J.-J., Mora, J., Lama, H., \& Molina, A. (2014). Evaluación dinámica de procesos lectores [Dynamic assessment of reading processes]. Madrid, España: Editorial EOS.

Preiss, D. (2009) The Chilean instructional pattern for the teaching of language: A video-survey study based on a national program for the assessment of teaching. Learning and Individual Differences, 19(1), 1-11. http://dx.doi.org/10.1016/j.lindif.2008.08.004

Preiss, D., Calcagni, E., Espinoza, A., Gómez, D., Grau, V., Guzmán, V., ... Volante, P. (2014). Buenas Prácticas Pedagógicas Observadas en el Aula de Segundo Ciclo Básico en Chile [Good pedagogical practices observed in the classroom of the second basic stage in Chile]. Psykhe, 23(2). http://dx.doi.org/10.7764/psykhe.23.2.716

Resing, W. (2013). Dynamic testing and individualized instruction: Helpful in cognitive education? Journal of Cognitive Education and Psychology, 12(1), 81-95. http://dx.doi.org/10.1891/1945-8959.12.1.81
Resing, W., \& Elliott, J. G. (2011). Dynamic testing with tangible electronics: Measuring children's change in strategy use with a series completion task. British Journal of Educational Psychology, 81, http://dx.doi.org/10.1348/2044-8279.002006.

Resing, W., Tunteler, E., de Jong, F., \& Bosma, T. (2009). Dynamic testing in indigenous and ethnic minority children. Learning and Individual Differences, 19(4), 445-450. http://dx.doi.org/10.1016/j.lindif.2009.03.006

Roskos, K.A., Moe, J.R., \& Rosemary, C. (2017). An Analysis of Implementation Strategies in a School-Wide Vocabulary Intervention. Journal of Education and Training Studies, 5(5), 184-196. https://doi.org/10.11114/jets.v5i5.2379

Rueda, M.I. (2013). La intervención en las dificultades lectoescritoras desde un enfoque multidimensional The intervention in the lecto-writing difficulties from a multidimensional approach]. Aula Abierta 41(1), 27-38.

Sánchez, E. (2010). La lectura en el aula. Qué se hace, qué se debe bacer y qué se puede hacer [The reading in the classroom. What is done, what should be done and what can be done]. Barcelona, España: Graò.

Schibli, K., Wong, K., Hedayati, N., \& D’Angiulli, A. (2017). Attending, learning, and socioeconomic disadvantage: Developmental cognitive and social neuroscience of resilience and vulnerability. Annals of the New York Academy of Sciences, 1396, 19-38. http://dx.doi.org/10.1111/nyas.13369

Souvignier, E., \& Mokhlesgerami, J. (2006). Using self-regulation as a framework for implementing strategy instruction to foster reading comprehension. Learning and Instruction, 16 (1), 57-71. http://dx.doi.org/10.1016/j.learninstruc.2005.12.006

Sternberg, R.J., \& Grigorenko, E.L. (2002). Dynamic testing. The nature and measurement of learning potential. Cambridge: Cambridge University Press.

Thiede, K. W., Griffin, T. D., Wiley, J., y Redford, J. S. (2009). Metacognitive monitoring during and after reading. In D. J. Hacker, J. Dunlosky, y A. C. Graesser (eds.), Handbook of metacognition and self-regulated learning (pp. 85-106). Mahwah, NJ: Lawrence Erlbaum.

Urquijo, S., García-Coni, A., \& Fernandes, D. (2015). Relación entre aprendizaje de la lectura y nivel socioeconómico en niños argentinos [Relationship between reading and socioeconomic level in Argentine children]. Avances en Psicología Latinoamericana, 33(02), 303-318. http://dx.doi.org/10.12804/apl33.02.2015.09

Valls, R., Soler, M., \& Flecha, R. (2008). Lectura dialógica: interacciones que mejoran y aceleran la lectura [Dialogical reading: interactions that improve and accelerate reading]. Revista Iberoamericana de Educación, 46, pp. 71-87.

van Driel, S., Slot, E., \& Bakker, A. (2018). A Primary Teacher Learning to Use Scaffolding Strategies to Support Pupils' Scientific Language Development. European Journal of STEM Education, 3(2), 05. https://doi.org/10.20897/ejsteme/3115 